D. REMAUD

ITECH

8, quai de Bir Hakeim 94410 Saint-Maurice

\title{
Développements récents en modélisation aux éléments finis 3D : apports pour l'ingénierie géotechnique
}

On décrit les évolutions récentes en ingénierie logicielle autour de la méthode des éléments finis et leurs applications pour la modélisation tridimensionnelle des ouvrages de géotechnique. Outils de définition géométrique, nouveaux mailleurs tétraédriques, algorithmes de résolution plus puissants, chacune de ces avancées apporte un confort d'utilisation et oriente l'utilisateur vers une meilleure approche de la modélisation. On montre ainsi que l'usage de calculs par éléments finis tridimensionnels devient compatible avec les contraintes de temps d'ingénierie pour l'analyse de projets courants.

Mots-clés : méthode des éléments finis, modélisation 3D, maillages 3D, ouvrages géotechniques, CESAR-LCPC.

\section{Recent advances in 3D finite elements modeling: contributions for geotechnical engineering}

We first describe recent advances in software developments applied to the finite element method and their application to 3D models of geotechnical structures. CAD tools, new tetrahedron meshers, new powerful algorithms; each of these new

developments provides a more user-friendly environment and leads the user towards a better modelisation process. Finally, we show that the use of 3D finite element models is now fully admissible for current engineering projects. 


\section{Introduction}

L'usage des éléments finis (EF) en géotechnique est aujourd'hui largement répandu en ingénierie pour l'analyse des déformations et des stabilités d'ouvrage. Basés sur des hypothèses simplificatrices, les modèles EF restent majoritairement bidimensionnels et par conséquent limités à l'analyse des ouvrages « linéaires ». Pour autant, dans les zones urbaines, les ouvrages sont complexes et les interactions entre groupes de structures sont souvent influentes sur leur comportement global. Aussi l'introduction de la troisième dimension est inéluctable dans nombre de projets.

Jusqu'à récemment, les modélisations EF 3D demeuraient réservées à des projets d'envergure car on leur reprochait d'utiliser des maillages plus lâches que les modèles 2D, ainsi que des temps de calcul longs, incompatibles avec les exigences temporelles de l'ingénierie.

Aujourd'hui ces constats peuvent être facilement battus en brèche avec le développement de machines performantes et d'outils de modélisation puissants.

A travers cet article, on s'attache ainsi à montrer l'apport derécents développements pour l'ingénierie géotechnique, en prenant pour illustration des exemples de modélisation réalisés avec CESAR-LCPC, code aux éléments finis développé par l'IFFSTAR (ex. LCPC, Laboratoire Central des Ponts et Chaussées) et ITECH.

Précision : Les exemples d'études réalisées avec les versions antérieures de l'outil, présentés dans le corps de l'article, ont pour but d'illustrer les avancées d'un point de vue qualité de la modélisation. L'auteur ne cherche pas à remettre en cause les résultats et leur interprétation à l'époque de leur réalisation. Aussi nous n'aborderons pas cet aspect.

\section{Pourquoi modéliser avec les éléments finis 3D?}

Tout projet de calcul d'ouvrage géotechnique est de nature 3D. Pour se ramener 2D, des hypothèses simplificatrices sont couramment employées : déformations planes ou axisymétrie.

La modélisation en déformation plane consiste à mener les calculs dans un plan, défini par une normale. Les déformations sont indépendantes de la coordonnée $z$ et le déplacement dans cette direction est nul. Ce type de modélisation est possible si la géométrie varie peu dans tous les plans parallèles au plan de déformation plane; de même pour les conditions aux limites, les conditions initiales et les chargements. Ce type de modélisation est répandu pour les calculs des semelles, des talus et remblais, des tunnels.

La modélisation en axisymétrie n'est, elle, applicable que si les caractéristiques du calcul (géométrie, conditions aux limites, conditions initiales et chargements) présentent une symétrie de révolution. Cette approche peut être appliquée aux fondations circulaires, aux galeries de tunnel circulaires, aux puits.

Mestat et al. (2005) décrivant ces approches ont aussi établi leurs limites. On retiendra que :
- les couches de sols sont rarement linéaires ou de révolution;

- les inclusions ou les système de soutènement ne respectent pas, en général, les conditions de planéité.

Il est cependant admis que les résultats des calculs plans sont souvent du côté de la sécurité en amplifiant les mouvements du sol.

Aussi-doit on procéder à une modélisation tridimensionnelle pour une représentation réaliste et non sujette à des hypothèses simplificatrices. La première approche pour le passage du 2D au 3D est l'extrusion, qui consiste à construire un maillage 3D par translation d'un maillage 2D. Cette méthode est régulièrement employée pour modéliser des fondations, on opère alors une extrusion verticale ou horizontale. En ingénierie du tunnel, le caractère tridimensionnel de l'excavation au front de taille est souvent modélisé en 2D par l'introduction d'un coefficient de déconfinement (méthode convergence-confinement). Pour prendre en compte des phénomènes plus complexes (soutènements, avancement d'un tunnelier, voûte parapluie...), les modèles de tunnel 3D sont réalisés à partir d'une représentation plane de la section à laquelle on fait subir une extrusion horizontale.

Si l'extrusion nécessite des conditions particulières de géométrie de sol et d'ouvrage, elle permet cependant de modéliser des structures complexes (comme l'ouvrage représenté ci-dessous, modélisé par le LCPC, pour l'EDF, dans les années 1980) (Fig. 1).

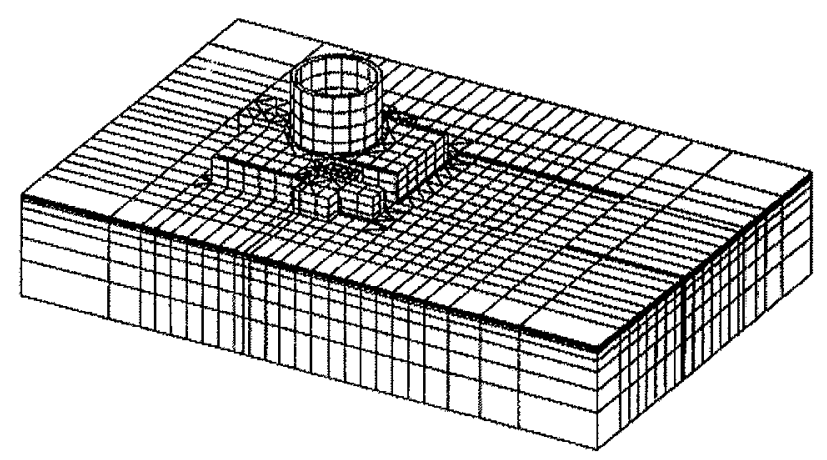

Fa. 1 Exemple de passage du 2D au 3D par extrusion.

Il a aussi souvent été reproché aux modèles 3D de ne pas avoir une densité de maillage cohérente avec celle généralement usitée en 2D. A densité équivalente, un modèle 3D génère des matrices de calcul plus grandes et en conséquence des temps de calcul plus longs. De plus, le codage 32-bits des systèmes est une autre limite à considérer. Cette limite ( physique ) induit en effet une limite numérique car les matrices de résolution ne peuvent pas être codées sur plus de $2^{31}$ octets.

Lors du passage du 2D au 3D, l'ingénieur devait donc faire des choix pour privilégier la cohérence géométrique et se focaliser sur un résultat. Ces alternatives sont : augmenter la taille des éléments finis ou privilégier l'interpolation linéaire au détriment de l'interpolation quadratique. Mestat et al. (2005) ont illustré ce constat sur la modélisation d'une galerie de tunnel (cf. Fig. 2). Sur ces deux modèles de projets distincts, on distingue nettement l'écart de densité de maillage. 

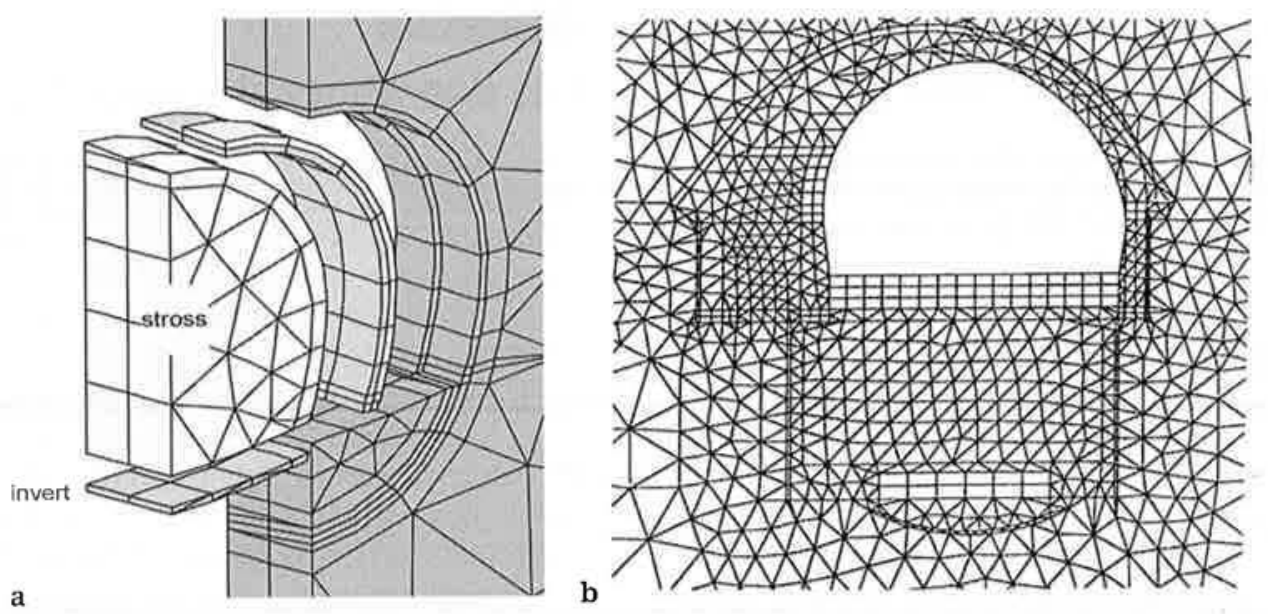

FG.2 Exemple de comparaison de densité d'éléments autour d'une cavité : a) pour un modèle $3 \mathrm{D} ; \mathrm{b})$ pour un modèle $2 \mathrm{D}$ en déformation plane (calculs réalisés avec CESAR-LCPC), d'après Mestat et al. (2005).

Les constructeurs d'ordinateurs proposent aujourd'hui des systèmes 64-bits couplés à d'importantes tailles de mémoires vives. La nouvelle version de CESAR-LCPC est adaptée à ces nouveaux environnements de travail. Aussi les limites sont repoussées et on peut raisonnablement appliquer aux modèles 3D des densités conséquentes pour des temps de résolution compatibles avec les contraintes de l'ingénierie.

\section{3}

\section{Évolutions de l'outil CESAR-LCPC}

\section{1}

\section{Une DAO intégrée}

L'ingénierie d'un projet passe par une première phase de projection en DAO (Dessin Assisté par Ordinateur). Ce travail préliminaire est important pour mettre en plan d'exécution les dispositifs de construction et les méthodes retenus. De plus, la récupération de la topographie du terrain au format numérique (MNT) est maintenant généralisée. Aussi intégrer les données de cette étape à la modélisation aux éléments finis tridimensionnelle est essentiel pour gagner du temps et accéder à un niveau de détail initial le plus proche possible du projet architectural. Cela se fait par les fichiers d'échange aux formats DXF, IGES ou STEP, standards aujourd'hui répandus en ingénierie logicielle pour la construction.

Ces données peuvent être exploitées dans CESARLCPC grâce aux derniers outils de la version 5 : import de fichiers DAO, surfaces NURBS (Non-Uniform Rational Basis Spline), intersections de volumes, génération de volumes complexes.
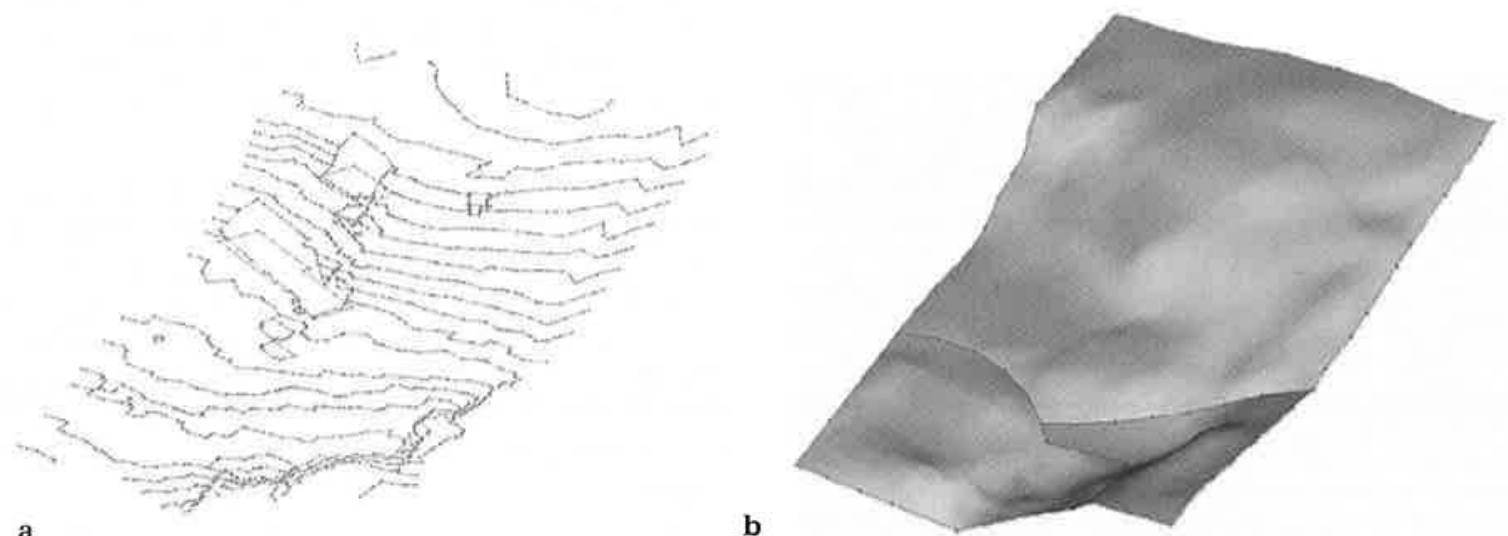

FIG.3 Import du modèle numérique de terrain et transformation en surface NURBS. Ainsi la DAO est de mieux en mieux intégrée, de plus en plus fusionnée dans les logiciels de modélisation évitant des allers-retours entre modélisateurs et projeteurs, potentiellement générateurs d'erreurs ou de pertes de données. 


\section{De nouveaux outils de maillage}

Dans les précédentes versions de CESAR-LCPC, le modélisateur générait les maillages par extrusion (extension d'un maillage 2D dans une direction perpendiculaire, horizontale pour les tunnels ou talus, verticale pour les fondations ou excavations). Une méthode basée sur la géométrie dite « super-éléments ») permettait de traiter certaines configurations complexes (intersection de galeries par exemple).

Dans la nouvelle version 5 de CESAR-LCPC, les méthodes précédentes sont conservées. On leur a adjoint le maillage en tétraèdres. Ce type d'éléments est utilisé de longue date en génie mécanique. Ils n'avaient pas rencontré jusqu'à présent un écho très favorable en ingénierie logicielle pour la géotechnique car ils sont très consommateurs d'éléments et de nœuds, ce qui est très pénalisant pour les calculs non linéaires. En effet, leur principe de fonctionnement est de mailler les surfaces délimitant le volume, puis de remplir le volume à partir de ces densités. Aussi l'utilisateur peut difficilement contrôler le nombre d'éléments générés.

En association avec les fonctionnalités présentées dans le paragraphe précédent, le mailleur tétraédrique automatique, implanté dans CESAR-LCPC, permet de s'affranchir des complexités géométriques. De plus, des éléments de barres spéciaux ont été développés pour modéliser des renforcements de sols (tirants d'ancrage, boulons ou pieux). Le mailleur tétraédrique ayant été conçu pour ( s'accrocher ) à ces éléments, on peut facilement modéliser les volumes de sols et les éléments structuraux les pénétrant, sans avoir à discrétiser la géométrie comme par le passé.

Enfin, pour assister l'utilisateur, un facteur de qualité a été associé au procédé de remplissage. Cet outil permet, d'une part, de jouer sur le nombre de nouds généré par le mailleur, et, d'autre part, de vérifier la densité des éléments, notamment dans les zones de fortes contraintes (cf. Fig. 4).

Ainsi le modélisateur ne travaille pas en boîte noire et peut à tout moment adapter son modèle à ses exigences de qualité et de résultat.

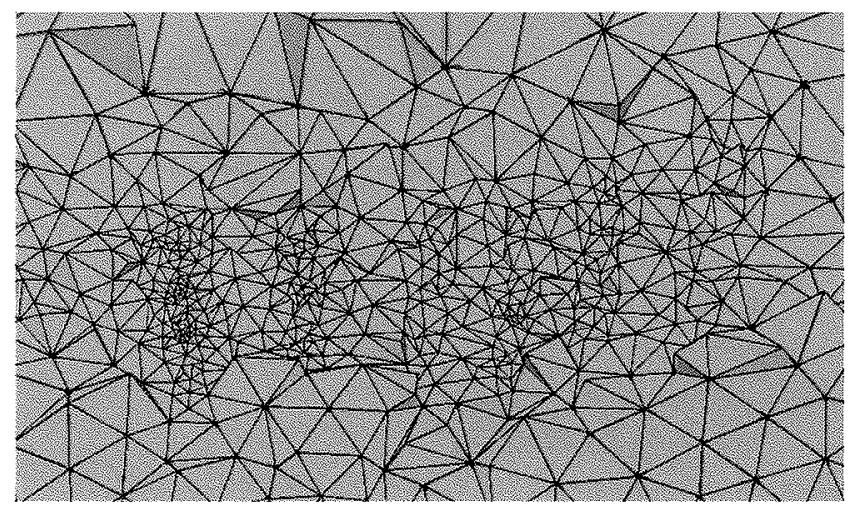

HG.4 Coupe dans un modèle 3D illustrant le remplissage tétraédrique autour d'éléments ancrés (tirants).

\section{Des algorithmes plus puissants}

L'optimisation des algorithmes de construction a été entreprise par l'IFSTTAR depuis plusieurs années (Rigobert, 2005). Ces travaux ont permis d'implanter un code pour matrices creuses fondé sur la méthode de Gauss multifrontale pour la résolution d'un système linéaire.

L'influence est immédiate sur le calcul des projets. On a relevé une réduction des temps de factorisation et de calcul par un facteur minimum de 10. Les temps de calcul des modèles 3D sont donc maintenant comparables à ceux des modèles $2 \mathrm{D}$ des versions antérieures.

Depuis l'introduction de cette nouvelle méthode, les environnements 64-bits sont apparus sur le marché des ordinateurs grand public ; ils sont donc accessibles aux bureaux d'études. Les gains précédemment établis sont amplifiés par la possibilité d'utiliser des tailles de mémoires vives conséquentes.

\section{:3.}

\section{Procédures d'analyse des coefficients de sécurité}

La définition d'un facteur de sécurité pour un ouvrage géotechnique ne va pas de soi. On peut proposer au moins deux approches : l'une porte sur les caractéristiques de résistance des sols, l'autre sur l'intensité du chargement.

Dans le cadre de la procédure de « réduction c-phi », cette sécurité est estimée sur la base d'un critère très simple : le facteur de sécurité est le plus grand nombre par lequel on peut diviser les caractéristiques de résistance (c et tan $\varphi$ pour le critère de Mohr-Coulomb) et obtenir un champ de contraintes compatible à la fois avec les chargements appliqués (considérés comme fixés) et la résistance des différents matériaux en jeu. L'autre approche de la sécurité consiste à estimer le facteur par lequel on peut multiplier un chargement sans atteindre la rupture (pour des caractéristiques de résistance données).

Ces approches intégrées dans la nouvelle version de CESAR-LCPC, permettent d'accéder aux états limites ultimes, ELU, des modèles non linéaires. Couplées à l'application de coefficients partiels par l'utilisateur sur les données du modèle, ces nouveaux outils permettront de reproduire les approches préconisées par les Eurocodes 7.

Pour valider le fonctionnement de la procédure de réduction (c c-phi ») dans CESAR-LCPC, une étude paramétrique a été réalisée sur un modèle $3 \mathrm{D}$ avec variation du ratio des cohésions non drainées $\left(c_{u 2}\right.$ ' $\mathrm{C}_{\mathrm{u1}}$ ), reprenant les caractéristiques du modèle de talus utilisé par Griffiths (1999) : géométrie paramétrée (cf. Fig. 5), couche de matériaux de faible caractéristique $\left(c_{u 2}\right)$ insérée dans un massif homogène $\left(c_{u 1}\right)$, critère de Tresca.

Les facteurs de sécurité calculés par CESAR-LCPC, sont en très bon accord avec les résultats de Griffiths (1999). Pour illustrer cet état, on présente l'état déformé du modèle 3D (cf. Fig. 6) calculé avec le ratio $\mathrm{C}_{\mathrm{u} 2} / \mathrm{C}_{\mathrm{u}: 1}$ de 0,33 , menant à un facteur de sécurité de 0,82 . 


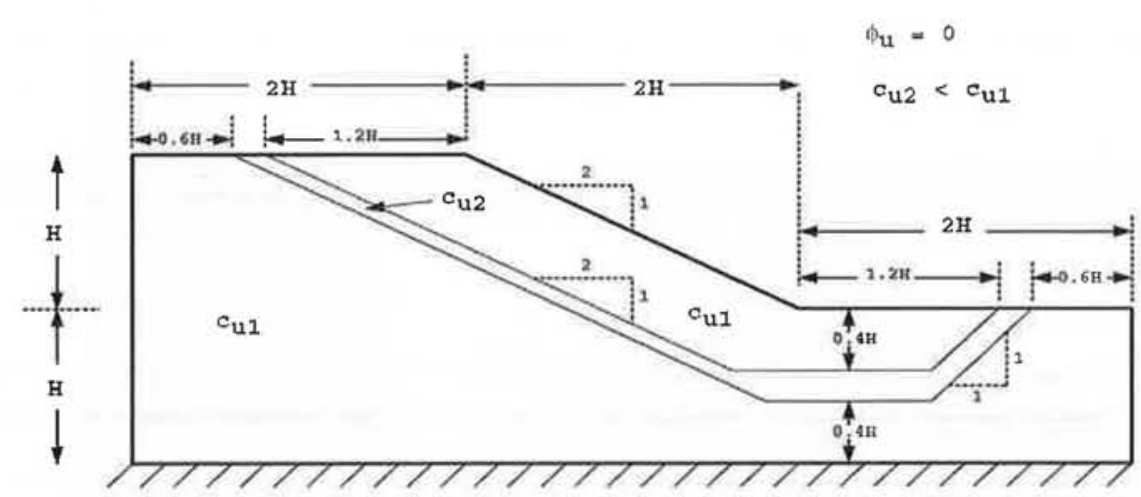

FIG.5 Géométrie du modèle de talus utilisé pour l'étude paramétrique de la procédure de réduction c-phi (Griffiths 1999).

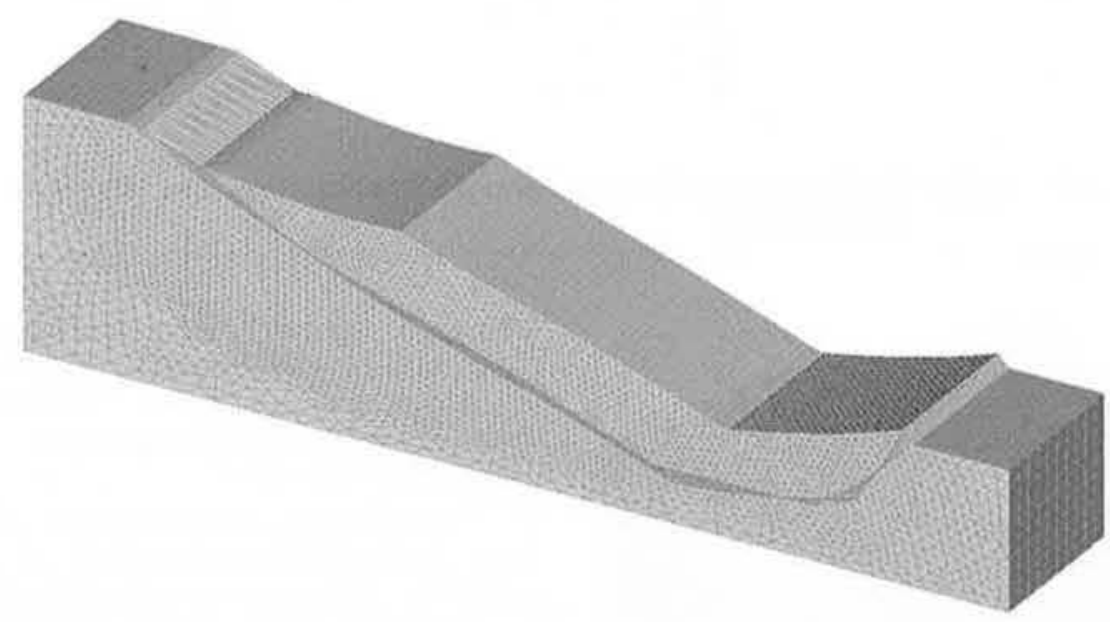

FiG.6 Déformée du modèle de talus $3 \mathrm{D}$ après calcul du facteur de sécurité par analyse c-phi $\left(\mathrm{c}_{\mathrm{u} 2} / \mathrm{c}_{\mathrm{u} 1}=0,33-\mathrm{FOS}=0,85\right)$.

4

\section{Apports des évolutions pour l'ingénierie géotechnique}

\section{1}

\section{Détails géométriques}

Récemment dans le cadre de l'analyse des fondations de la tour Odéon à Monaco, Terrasol a exploité les fichiers DXF 3D du bureau d'étude en phase exécution : topographie numérique du terrain, systèmes de soutènements.

Les surfaces des couches de sols ont ainsi été positionnées au plus juste à partir des données du terrain et l'ouvrage est implanté de façon précise par rapport à son environnement géologique et au bâti existant. Ce niveau de détail était primordial dans le cadre du projet de la tour Odéon puisque des contraintes importantes étaient imposées par la maîtrise d'ouvrage pour que les travaux n'impactent pas les bâtiments environnants. Ainsi les tassements calculés ne devaient pas dépasser quelques millimètres.
Par ailleurs, le modèle en éléments finis 3D réalisé avec CESAR-LCPC avait aussi pour objectif de valider certaines hypothèses de soutènement, l'intégration précise des tirants était donc essentielle dans ce contexte. En illustration, on montre l'implantation des ancrages et soutènements (cf. Fig. 7) ainsi qu'une vue du modèle complet (cf. Fig. 8).

On retiendra aussi pour ce projet que les temps de calcul sont de l'ordre de $18 \mathrm{~h}$ sur une machine grand public (Windows 64 bits, 12 Go de RAM). Étant donné la complexité du modèle et le nombre de phases de calcul (57), cette durée était admissible pour le bureau d'études et elle a autorisé l'intervention pour modifier le modèle (paramètres des matériaux, cas de charges...) et le passage de calcul de stabilité (procédure de réduction c-phi).

Les résultats des calculs ont entraîné des modifications du système de soutènement réalisé sur la zone du Collège et validé les principes de fonctionnement globaux. 

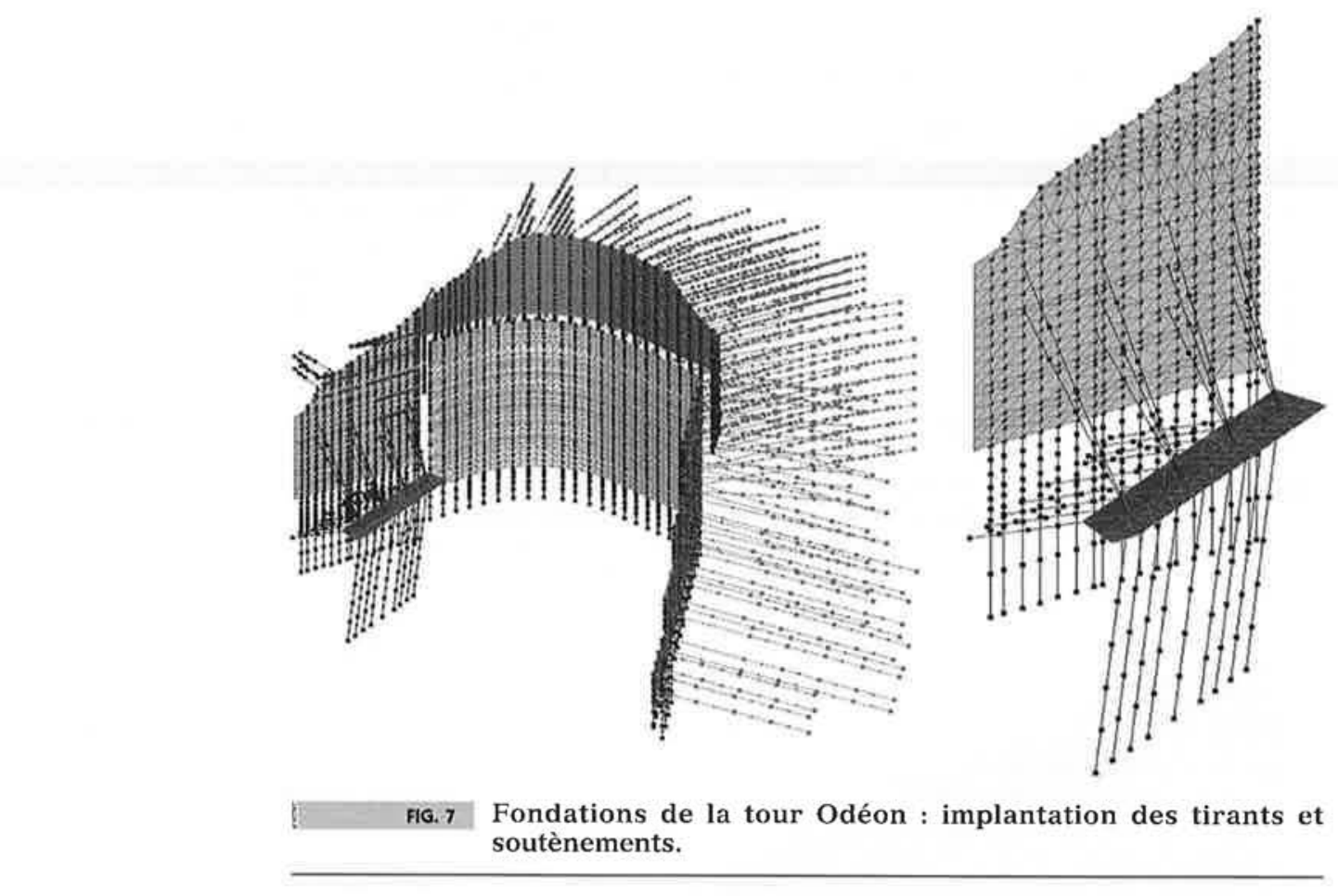

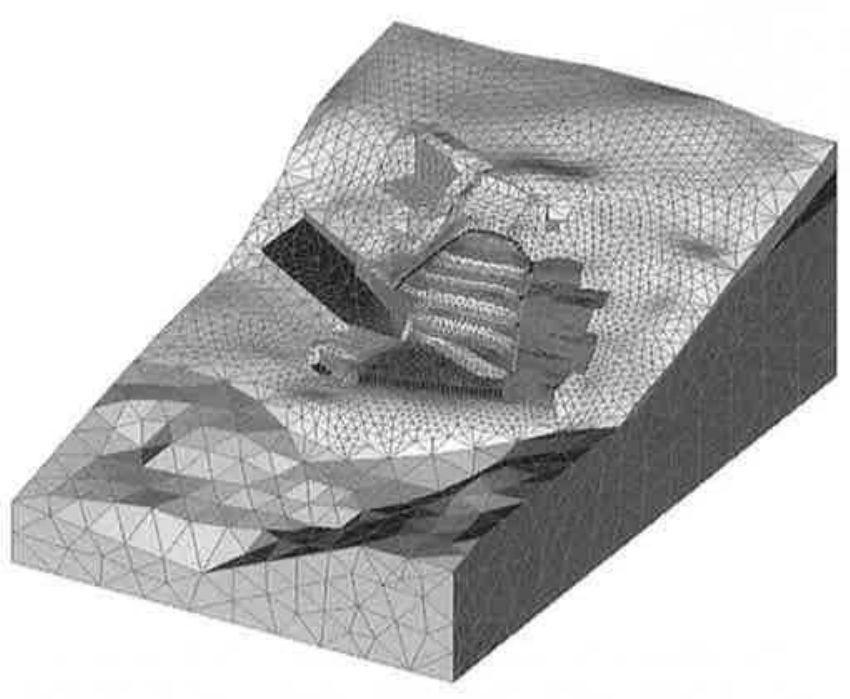

FIG. 8 Excavation et fondations de la tour Odéon à Monaco : vue complète du maillage.

\section{2}

\section{Qualité de maillage 3D}

Nous illustrons l'évolution de la qualité des maillages 3D par l'analyse a posteriori de deux modèles réalisés sur les versions précédentes de CESAR-LCPC.

Le premier exemple est un projet réalisé par Fondasol pour modéliser la réhabilitation du quai de l’Europe (étude réalisée en 2004). Utilisant les outils à sa disposition, l'ingénieur a utilisé la méthodologie de maillage en super-éléments ; cela a nécessité de décomposer le modèle géométrique en un grand nombre de régions volumiques permettant par la suite un maillage en super-éléments (hexaèdres, pentaèdres). Pour l'intégration des éléments de structures notamment les ancrages arrière, on a aussi dû adapter la géométrie pour qu'elle " accroche » les futurs éléments finis de type barres. Si cette méthode autorise la modélisation de tout type d'ouvrage 3D, elle contraint à un redé- coupage géométrique souvent surabondant. De plus, l'usage des super-éléments impose la propagation des découpages d'un élément à son voisin ; aux limites du modèle, on retrouve alors des densités de maillage non souhaitées et inutiles.

En utilisant les évolutions des outils géométriques et le mailleur tétraédrique intégré, il serait aujourd’hui possible : 1) de représenter l'ensemble des détails de la géométrie du projet ; 2) de générer les volumes nécessaires à la modélisation (avec ou sans intersections) ; 3) de mailler les régions volumiques ; puis finalement, 4) d'accrocher automatiquement les éléments volumiques aux éléments de type barre (modélisation des tirants d'ancrages).

Ces évolutions sont illustrées sur les figures 9a (modèle original) et $9 \mathrm{~b}$ (modèle réinterprété).

On constate une simplification notable de la géométrie. La qualité du maillage est aussi nettement améliorée : il n'y a pas d'éléments finis en surnombre aux limites du modèle et l'interpolation des éléments est passée de linéaire à quadratique, à nombre de nœuds identiques.

Nous avons analysé avec le même recul le maillage 3D réalisé par Terrasol pour la fondation du viaduc de la Grande Ravine à La Réunion. Géométriquement, ce projet présentait des difficultés : il s'agissait d'une fondation cylindrique dans une stratigraphie de schistes à double pente.

En 2003, l'ingénieur en charge du projet de calcul a réalisé le modèle en combinant deux méthodes de maillage : utilisation des super-éléments pour la culée, extrusion par extensions horizontales et verticales du maillage. Devant représenter l'interaction sol-structure par des éléments d'interfaces (surfaces de contact), le modélisateur a aussi dû utiliser des éléments finis à interpolation quadratique. Cette procédure augmentant le nombre de nœuds, la densité de maillage a été choisie pour générer des tailles de matrices de résolution compatibles avec l'environnement de calcul de l'époque (Windows 32-bits) et calculables en un temps compatible avec le projet (Fig. 10). 


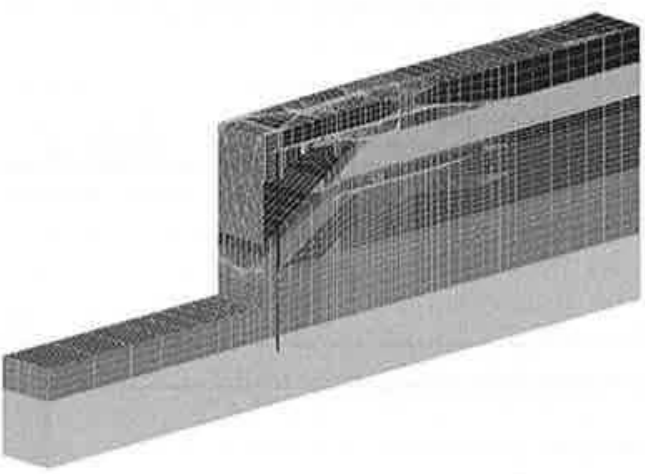

a)

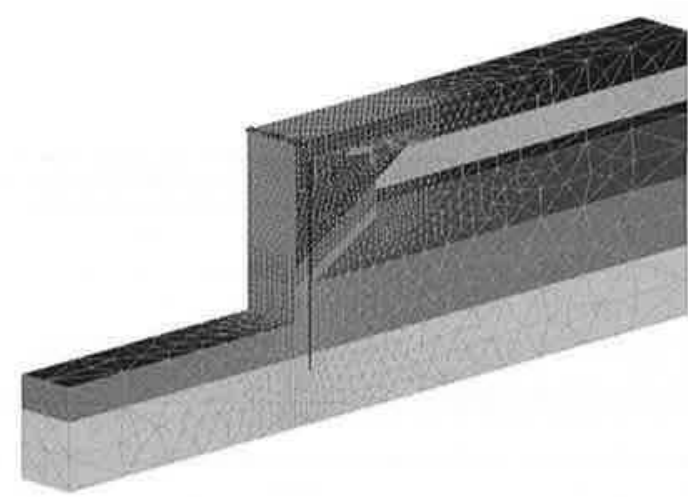

b)

FIG.9 Quai de l'Europe par Fondasol : réinterprétation du modèle version 4 réalisé en 2004 (a) avec la version 5 (b).

Réinterprétant aujourd'hui ce modèle avec la nouvelle version de CESAR-LCPC, les points suivants pourraient être améliorés : 1) représentation géométrique de la stratigraphie des sols (import de MNT, utilisation des surfaces NURBS) ; 2) variation progressive de la taille des éléments de la culée vers les limites extérieures du modèle (maillage tétraédrique) ; 3) densité de maillage plus conséquente à proximité de la culée, endroit des fortes sollicitations.

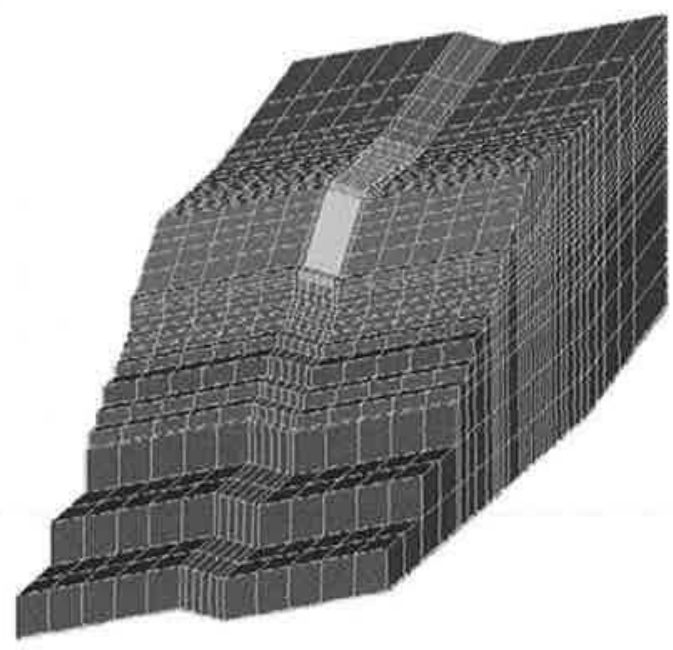

Les modèles réalisés par nos soins montrent qu'on peut facilement reproduire le modèle (Figs. 10 et 11), mais surtout que la densité de nœuds à proximité de la fondation peut être significativement et simplement augmentée.

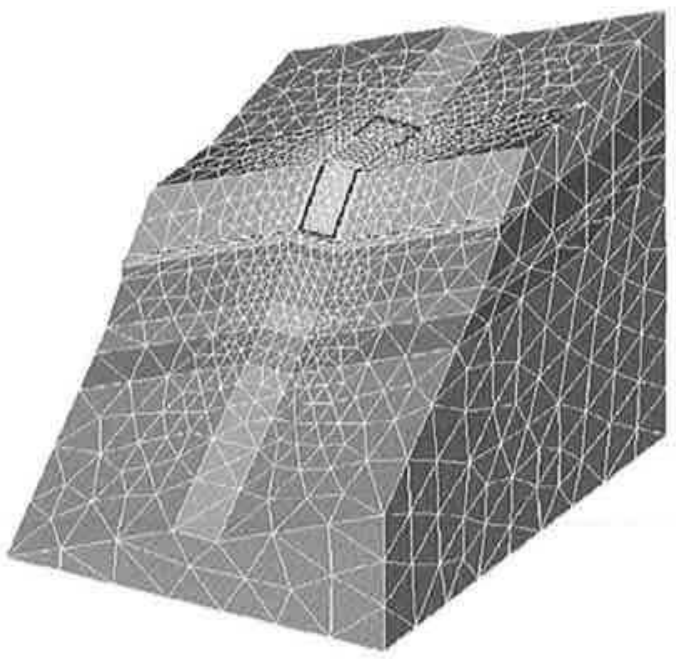

FG. 10 Zoom sur la modélisation de la curée, en version 4 , en version 5 avec nombre de nœuds équivalent; en version 5 avec nombre de nœuds triplé.
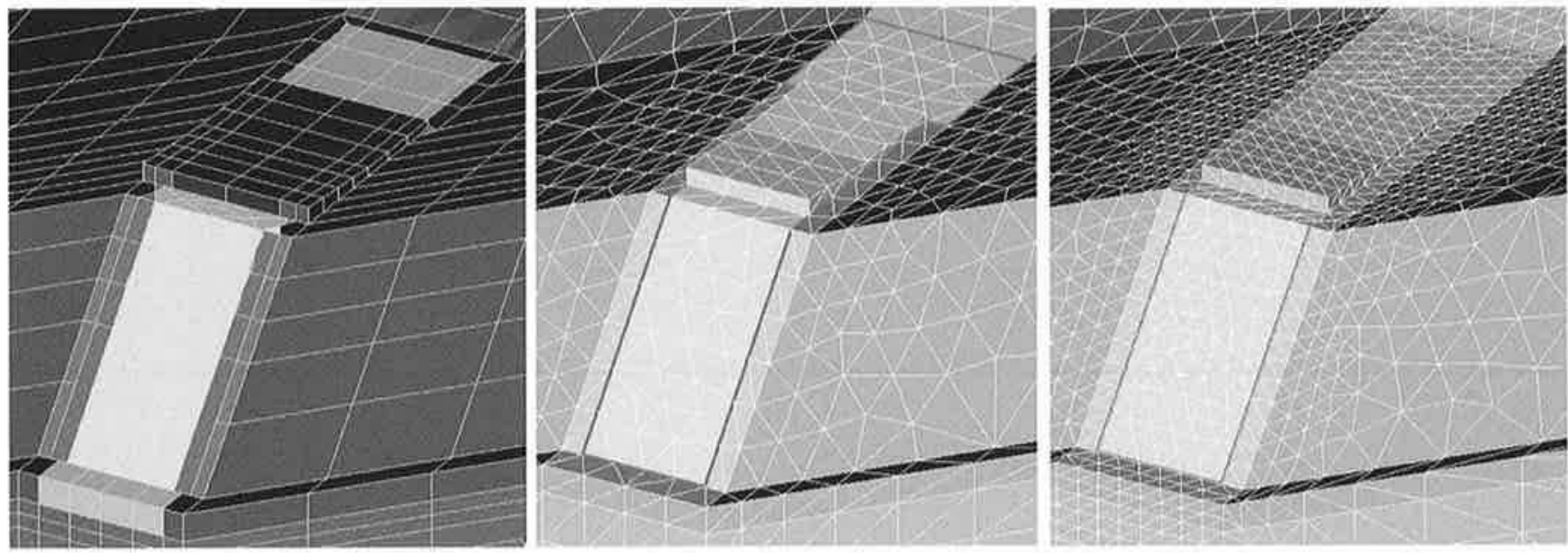

FIG. 11 Viaduc de la Grande Ravine par Terrasol : réinterprétation du modèle version 4 réalisé en 2003 (à gauche) avec la version 5 (à droite). 
5

\section{Conclusion}

Les évolutions tant du solveur et des pré- et post-processeurs présentées dans cet article permettent maintenant d'aborder la modélisation 3D de façon courante. La modélisation de la géométrie des ouvrages grâce à des mailleurs performants est de plus en plus précise. Les environnements de travail et les algorithmes de résolution apportent une puissance de calcul décuplée. Aussi l'ingénieur a maintenant à sa disposition des outils permettant de traiter des aspects jusqu'alors peu abordés dans les projets 3D (calculs de stabilité, coefficients de sécurité, études paramétriques).
Cette nouvelle offre va aussi évoluer fortement avec l'utilisation optimale des nouveaux environnements de travail codés 64-bits, encore peu employés car récemment apparus sur le marché ( grand public ). Aussi les efforts de l'IFSTTAR et de l'ITECH sont maintenus pour offrir à l'utilisateur un outil CESAR-LCPC pérenne et intégrant les résultats de la recherche. Ainsi la future version 6 (courant 2014) permettra une interactivité accrue avec l'utilisateur pour une modélisation simple, souple et performante (parallélisation des calculs). Elle intégrera, entre autres développements, un outil de construction de lois de comportement (y compris HSM).

\section{Bibliographie}

Griffiths D.V., Lane P.A. - Slope stability analysis by finite elements. Géotechnique $49, \mathrm{n}^{\circ} 3,1999$, p. $387-403$

Mestat P., et al. - Performances des modèles d'éléments finis 3D en géo- technique. Proc. XVIth ICSMGE, Osaka, 2005, p. 1091-1094.

Rigobert S. - Implantation dans CESARLCPC d'un schéma multifrontal pour la résolution de systèmes linéaires de grande taille. Bulletin des Laboratoires des Ponts et Chaussées, $n^{\circ} 256-257,2005$, p. 39-51.

ITECH, IFSTTAR - CESAR-LCPC CLEO3D, Manuel d'utilisation, $259 \mathrm{p}$. 